\title{
The Overarching Concept of Salutogenesis in the Context of Health Care
}

\author{
Geir Arild Espnes, Unni Karin Moksnes, \\ and Gørill Haugan
}

\section{Abstract}

Two concepts that widely impact on our ways to work with health is health promotion and salutogenesis (For a quick overview of the concept of salutogenesis, read Lindström B. \& Eriksson M. (2010). The Hitchhiker's Guide to Salutogenesis. Folkhälsan Research Center). The concept of health promotion was voted for

G. A. Espnes $(\triangle)$

Department of Public Health and Nursing, NTNU Norwegian University of Science and Technology, Trondheim, Norway

NTNU-Center for Health Promotion Research, Trondheim, Norway

e-mail: geirae@ntnu.no, geir.arild.espnes@ntnu.no

U. K. Moksnes

Department of Public Health and Nursing, NTNU

Norwegian University of Science and Technology,

Trondheim, Norway

NTNU-Center for Health Promotion Research,

Trondheim, Norway

Faculty of Nursing and Health Science,

Nord University, Levanger, Norway

e-mail: unni.moksnes@ntnu.no

G. Haugan

Department of Public Health and Nursing, NTNU

Norwegian University of Science and Technology,

Trondheim, Norway

Faculty of Nursing and Health Science,

Nord University, Levanger, Norway

e-mail: gorill.haugan@ntnu.no,

gorill.haugan@nord.no use by the participants of World Health Organization (WHO) general assembly in 1978. And after 8 years, the concept of health promotion was filled with content by the WHO meeting in Ottawa in 1986. Meanwhile, salutogenesis as a concept was constructed of the Israeli scientist Antonovsky during the 1970s. It can be said that both health promotion and salutogenesis grew out of a wanting to understand health development rather than understanding health as a variable tied to the presence or absence of disease developments. This chapter concentrates on discussing the use of the salutogenic framework on the understanding of health care situations.

\section{Keywords}

Health $\cdot$ Health promotion $\cdot$ Salutogenesis $\cdot$ Resources · Pathogenesis · Treatment

\subsection{Salutogenesis: Turning Health Concerns from Solely be Occupied with What Gives Disease to What Gives Health}

Salutogenesis has become a frequently used word or concept in the health domain, and especially within the public health and health promotion 
area (see [1]). But where does the word come from? And what does this concept mean? This chapter sets out to reveal the answer to both these questions and also to investigate how the understanding of health can be encompassed in health care and disease treatment.

The WHO Ottawa Charter [2] almost 35 years ago clearly defined that health is ".... a resource for everyday life ... A positive concept emphasizing social and personal resources, as well as physical capacities ... To reach a state of complete physical, mental and social well-being." This explanation of what health is gave a whole new understanding of the rationale for what brings health instead of the sole pursuit of the reasons of a disease or how to prevent diseases. These two equally old concepts of health promotion and salutogenesis sometimes are deemed the "starting shot" for the new challenge of enhancing health rather than explaining and preventing disease. A new aera had begun.

Salutogenesis was first used as a concept of health by the Israeli medical sociologist Aron Antonovsky. In the 1960s, Antonovsky studied female survivors from the Second World War's German concentration camps who by then had become grandmothers. What he found was remarkable. A number of the Jewish grandmothers, now living in Israel, had not only survived the concentration camps, but also been able to live a good flourishing life, with good mental and physical health, in spite of the horrors in the camps. Antonovsky stated that even if only a few would have lived through the horrors and still were able to live a flourishing life that would be most remarkable and should be subject to thorough studies in search for the overarching question; what is the origin of good health? One of Antonovsky's deviations from pathogenesis was to reject the dichotomization into categories of diseased or healthy. Antonovsky stated that disease, stressors, and unpredictability are part of life and can never be controlled completely. The interesting question that came to his mind was: how can we survive in spite of all this? The answer to this was understood as the individual's sense of coherence and ability to identify and use generalized and specific resistance resources.

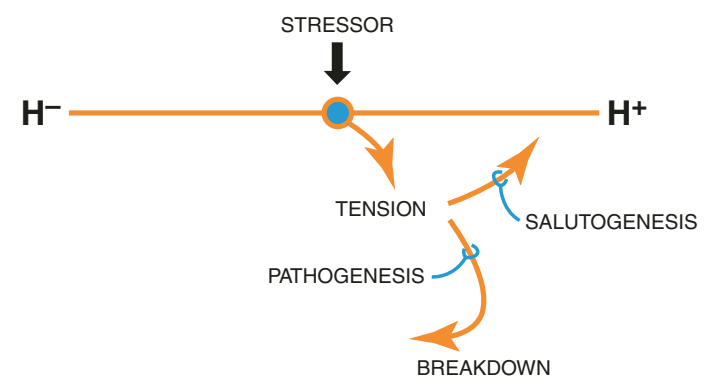

Fig. 2.1 The ease dis-ease continuum. (Published with permission from Folkhälsan Research Center, Helsinki, Lindström \& Eriksson [3])

One of the keys to the salutogenic approach was to describe health as a continuum between "total health $(\mathrm{H}+)$ and total absence of health" $(\mathrm{H}-)$ or the "ease - dis-ease continuum" (see below Fig. 2.1).

At any time, each of us can be placed on this continuum [4]. Stressors can upset our position, and we come under tension. Here are two options: either the pathogenic forces overtake us and we break down or we regain our health through SOC and move toward $\mathrm{H}+$. The important point is to focus on what moves an individual toward the ease pole of the continuum, regardless of where the individual is initially located. By the continuation of his studies, Antonovsky presented a few distinct characteristics of what gave good health to people, as well as developed a new health theory of "salutogenesis." The name of "salutogenesis" was constructed by combining the Latin word Salut (health - or to your health) with the Greek word Genesis (origin). Salutogenesis has become an interesting concept for scientists and practitioners from a wide range of backgrounds who had been in search for an approach to study what brings health. Especially, the movement for health promotion research and work has shown great interest.

For many years, WHO had enquired for a reorientation in health care representing the use of both the resource (salutogenesis) and the treatment paradigms (pathogenesis) as complementary in health care. The health promotion approach had surfaced as an alternative to disease prevention, keeping health as a target rather than avoidance of disease on the WHO 1978 World 
Conference in Alma Ata. After 8 years of intensive work to establish an understanding of health promotion and the need for an alternative to disease prevention across the world, the Ottawa Charter on Health Promotion was launched in the WHO World Conference in Ottawa 1986 [2], and the health social anthropologist that had led the work became notoriously famous. Her name is Ilona Kickbusch.

The Ottawa Charter became the answer to the request for a reorientation of the world's health care systems. One can claim that there is quite an unrealized potential in health care to be more protective and promotive of health. However, the Ottawa Charter was in lack of a theoretical model or theoretical approach to back its ideas. Since salutogenesis was developed as a paradigm in opposition to the "pathogenic orientation which suffuses all western medical thinking" ([5], p. 13), it had to be considered a health promotion concept. In his brief 1996 paper in the journal Health Promotion International, Antonovsky challenged the health promotion field to adopt salutogenesis as a health promotion theory.

In principle, applying salutogenesis as a health theory in the health promotion field could mean to restrict the leading pathogenic orientation in health care practice (research and policy) and complement or change it by a salutogenic orientation in everyday practice and research. It also became evident that Antonovsky had come up with a theoretical basis for the concept of salutogenesis, and the underlying concept of "Sense of Coherence" which explains what brings good health. The next part of this chapter dives further into the key concepts of Antonovsky's salutogenic health model, namely sense of coherence and resistant resources.

\subsection{The Sense of Coherence and Resistant Resources}

As stated above, Antonovsky introduced the salutogenic model as a new possible paradigm for health research [5]. In a lecture at Berkley in 1993 [6], he defined the concept of salutogenesis as "the process of movement toward the health end of a health ease/dis-ease continuum" and defined the life orientation concept of sense of coherence (SOC) as follows:

$$
\begin{aligned}
& \text { "... a global orientation that expresses the extent to } \\
& \text { which one has a pervasive, enduring though } \\
& \text { dynamic feeling of confidence that one's internal } \\
& \text { and external environments are predictable and that } \\
& \text { there is a high probability that things will work out } \\
& \text { as well as can reasonably be expected." [7] }
\end{aligned}
$$

Accordingly, salutogenesis is understood to describe the process of enabling individuals, groups, organizations, and societies to emphasize on abilities, resources, capacities, competences, strengths, and forces in order to create a strong SOC; that is, to perceive life as comprehensible, manageable, and meaningful which represent three central components in SOC. Recent research shows this model is an effective approach to positive health development in a life course perspective [1]. However, the potential of this model has not been fully explored in health promotion practice and research [1].

The salutogenic model includes three central concepts: generalized resistance resources (GRRs), specific resistant resources (SRR), and the above-mentioned SOC. The GRRs are of both external and internal characters; people have at their disposal resources of both internal and external characters which make it easier for them to manage life [4]. Specific resistance resources, on the other hand, are optimized by societal action in which health promotion has a contributing role, for example, the provision of supportive social and physical environments [1].

The GRRs are of any character ranging from material to virtual and spiritual dimensions of the mind, processes, and psychological mechanisms. The main thing is that people are able to use the GRRs for their own good and for health development. The GRRs, characterized by underloadoverload balance and participation in shaping outcomes (empowering processes), provide a person with sets of meaningful and coherent life experiences, which in turn create a strong SOC [1].

While GRRs are the prerequisites for developing a strong SOC, the capability to use GRRs is based on people's SOC, a concept that has been 
shown to be of key importance in health research, correlating positively with good health outcomes, quality of life, and most psychological measures of well-being [1]. Today, there are hundreds of articles referring to the SOC in individuals and groups, as well as population studies that demonstrate the strength of this concept and phenomenon. Antonovsky stated that people's SOC is mainly developed in childhood and early adulthood. However, new research points to the fact that $\mathrm{SOC}$ is a continuous process throughout the entire life [1]. The perception of coherence is based on cognitive, behavioral, and motivational factors which are improved by raising the awareness of the population, empowering the population and engagement in areas which are meaningful to the population.

\subsection{Salutogenesis in Health Care Settings}

The health care sector is still primarily defined by a pathogenic paradigm, and the health care system is most often anticipated as the system of struggle against pathological developments, or as Antonovsky expressed it "health care or more correctly the disease care system" ([5], p. 12). The health care area has therefore often been seen as challenging for the application and implementation of a salutogenic approach. To understand the challenge completely, one needs to ask what is the essence in the challenge of integrating these newer, more modern, and comprehensive health perspectives into health care?

The health care sector intends to professionally manage illness by trying to prevent or cure diseases, or if this is not possible, at least to offer care for chronic patients and palliative care. However, the contribution of health promotion is still marginal in the health care sector. Reorientation of health services, as demanded by the Ottawa Charter [2], has not yet happened in accordance to the expectations [8,9]. There is still quite an unrealized potential in health care to be more protective and promotive of positive health. Further, also salutogenesis has quite an unrealized potential for being more evidence based in reference to preserving and promoting health in different patient groups living with disease and infirmity. In his 1996 publication [5], Antonovsky suggested that the appeal of the full salutogenic model for those engaged in health promotion cannot be on the grounds of powerfully demonstrated efficacy in producing significant health-related change outcomes. It can be understood that to release the unreleased potential of using the salutogenic framework in health care, the only way to test the potential and effectiveness is to start using it in different health care settings. A relevant question today is, therefore, how far has the change toward a more protective and promotive approach in health care come since Antonovsky wrote this statement, and are there any differences between different health care settings?

First things first. Salutogenesis - the newer and more focused concept-has been introduced by Antonovsky into health promotion, which represents an older and broader concept, field, and movement. As pointed to above, Antonovsky [5] underlined that "the basic flaw of the field (of health promotion) is that it has no theory." Thus, he proposed "the salutogenic orientation... as providing a direction and focus to this field." $\mathrm{He}$ also stated that "the salutogenic model is useful for all fields of health care. In its very spirit, however, it is particularly appropriate to health promotion." Hence, health promotion in health care definitely has the blessings of Antonovsky. Therefore, we have to clarify how the salutogenic orientation or model and its related construct of SOC can be integrated into health care, directly or via (re-)orienting health promotion in health care indirectly.

\subsection{What Can Salutogenesis Mean for Health Care, Across Settings?}

In health care settings, the salutogenic paradigm can be used for two purposes: either to guide health promotion interventions in health care practice across settings or to (re)orient health care research as such. For this, the salutogenic 
paradigm offers specific concepts, assumptions, and instruments. According to Pelikan [10], three quite different conceptual forms can be distinguished: (1) a salutogenic orientation, (2) a salutogenic model, and (3) the construct of the SOC and a methodologically sound way to operationalize it. These three forms first have to be specified in more detail, to be applied later to the whole field of health care and later for specific settings. For that, health care has to be understood as a complex of a strongly interrelated professional practice, with clinical research and supporting policy. Therefore, applying salutogenesis in health care successfully cannot just be done by introducing salutogenesis in health care practice; there is also a need for a change in underlying health care policy.

\subsubsection{The Salutogenic Orientation and Health Care}

The first and most broad form of salutogenesis, a salutogenic orientation, is described by three assumptions: (a) the human system is subject to unavoidable processes toward an unavoidable final death. Therefore, the necessity of adaptation or coping with accompanying tension that may result in stress is universal and not the exemption. (b) A continuum model, which sees each and all of us somewhere along a health ease/dis-ease continuum. Therefore, a dichotomization into healthy and sick is not very helpful. (c) The concept of salutary factors or health-promoting factors are shown to actively promote health, which represents better health rather than just being low on risk factors (see [5], p. 14). Therefore, both risk and salutary factors have to be attended.

From these three assumptions follow implications for health promotion. A salutogenic orientation as the basis for health promotion directs both research and action efforts: (1) to encompass all persons, wherever they are on the continuum, and (2) to focus on salutary factors, which (3) relate to all aspects of the person instead of focusing on a particular diagnostic category as in curative medicine or (even) in preventive medicine.
Applying these assumptions and implications to health care practice would mean, again according to Pelikan [10], that (1) since a salutogenic orientation encompasses all individuals independently of their position on the ease/dis-ease continuum, health care should not only just care for the health of its patients, but take responsibility for the health of its staff and the health of citizens as well (however, dichotomous classification of individuals into those who have some specific disease or not, is unavoidable for doing curative medicine on patients); (2) in relation to these three groups of patients, health care staff, and citizens, not only their risk factors have to be dealt with or fought by health care, but also possible health-promoting (salutary) factors have to be enhanced in curative, preventive, protective, and promotive practice; (3) a holistic approach, including all sides and aspects; physical, mental, spiritual/existential, and social aspects of a person have to be taken into account in dealing with all people affected by health care.

In principle, to apply these assumptions on health care sounds plausible and rational, but three aspects need to be fulfilled: firstly, to realize a policy change of the mandate of health care is necessary; secondly, to understand that the traditional diagnostic and therapeutic repertoire of health care has to be widened; and thirdly, a radical change of clinical understanding and application is implied. The last of these three might be especially difficult since part of the spectacular medical success rests on focusing on a narrow biomedical model.

\subsubsection{The Salutogenic Model}

A second way of understanding salutogenesis in relation to health care settings is to understand Antonovsky's specific and rather complex salutogenic model ([7], see Chap. 7)). Within this model, the concept of GRRs is introduced as "a property of a person, a collective or a situation which, as evidence or logic has indicated, facilitated successful coping with the inherent stressors of human existence" ([5], p. 15). This model has not been further explored, even if major psy- 
chosocial, genetic, and constitutional GRRs are specified within this model [11]. There is, however, a possibility via scientific scrutinization of a wider view of this complex model encompassing large societies and a possibility to further explore the model as an underlying understanding for policy and society interventions.

In some countries, like Norway, we have through the last years seen a growing number of practical interventions in public health and health care that has been based in the general salutogenic model or other resource-based models [12]. It has also been observed how practical salutogenic work solutions have been used in health care among young in schools [13], for elderly both in nursing homes [14] and as an intervention approach in care situations among elderly outside nursing homes [15]. These new studies make their ways into health planning and health policy, but now also increasingly in care and health care. It might seem like the change in orientation that De Leeuw [8] was asking for slowly is appearing. How central researchers in the area of salutogenesis research sees the future developments is spelled out in two central publications: (1) the Handbook of Salutogenesis from 2016 [16] and (2) in the 2019 article entitled "Future directions for the concept of salutogenesis: a position article" [17].

\subsubsection{The Sense of Coherence and Health Care Settings}

If one utilizes the salutogenic model in health care (see above), the GRRs specified in detail in the salutogenic health model would have to be more adequately taken into account in health care practice and research, as well as in policy documents describing research activities and practices. The model and its implications make much sense for health care in different settings and afford a more holistic and complex outlook and a widening of diagnostic and therapeutic methods applied.

The third most focused form of salutogenesis, the specific construct of SOC which has been introduced as a central factor in the salutogenic model of health, is defined as: a generalized orientation toward the world which perceives it, on a continuum, as comprehensible, manageable and meaningful ([5], p. 15).

Antonovsky further stated that

The strength of one's SOC, I proposed, was a significant factor in facilitating the movement toward health. This construct answers what do all these GRRs have in common, why do they seem to work. What united them, it seemed to me, was that they all fostered repeated life experiences which, to put it at its simplest, helped one to see the world as 'making sense', cognitively, instrumentally and emotionally ([5], p. 15).

Here Antonovsky introduced the SOC as a moderator or mediator of other determinants of health rather than a specific determinant of health. "What matters is that one has had the life experiences which lead to a strong SOC; this in turn allows one to "reach out," in any given situation and apply the resources appropriate to that stressor. "The strength of one's SOC is shaped by three kinds of life experiences: consistency, underload-overload balance, and participation in socially valued decision making. The extent of such experiences is molded by one's position in the social structure and by one's culture.. ." ([5], p. 15).

Is there one pivotal argument of how the SOC can be introduced into health care? A thought experiment:

Being ill and becoming a patient in professional health care is often a rather threatening life experience for people and being a health care professional is a rather demanding job. Therefore, using the SOC concept for making the health care context and the culture as far as possible consistent, underload-overload balanced, and participatory for patients, health care staff, and visitors could be an adequate argument and way to make health care systems more salutogenic driven, generally. This is possible, since "social institutions in all but the most chaotic historical situations can be modified to some degree" ([5], p. 15). A different way to think about this, is to work with the possible feasibility, effectiveness, and efficiency in developing salutogenic "standards" [18] and make institutional contexts more salutogenic. Even if Antonovsky assumed that one's SOC cannot be radically transformed, he 
left it open that the SOC could be shaped and strengthened, so that it in turn can push people towards health [15]. Therefore, in reference of patients' situation, improving SOC by increasing their health literacy among an array of other coping resources could become an explicit goal of chronic disease management.

\subsection{Conclusions}

The salutogenic perspective has clearly a potential to be applied in the health care across settings in relation to health promoting interventions for the health of patients, staff, and citizens, and in supporting health-promoting structures and cultures of health care institutions for better everyday practice and policy.

As we have pointed to in this chapter, there are some very important implications for utilizing the salutogenic approach and model as a way to work in health care settings. The field or setting will need (1) to encompass all persons, wherever they are on the ease/(dis)ease continuum and (2) to focus on known salutary factors, which (3) relate to all aspects of the person rather than solely focusing on a particular diagnostic medical category or criteria.

There is, of course, as for most other concepts and theories, a clear need for further research, no doubt. Especially research focusing on the salutogenic model and on the specific role of SOC as a predictor, mediator, or moderator is needed. Moreover, research shaped to lead to a better conceptual clarity and application of more complex research designs, especially on the link between SOC and other aspects of health than subjective and mental health, as well as on the impact of health care setting functioning are required.

\section{Take Home Messages}

- The WHO Ottawa Charter clearly defines that health is a "...a resource for everyday life... A positive concept emphasizing social and personal resources, as well as physical capacities...."

- The Israeli medical sociologist Aron Antonovsky presented a few distinct characteristics of what gave good health to people, as well as developed a new health theory termed "salutogenesis." The name of "salutogenesis" was constructed by combining the Latin word Salut (health) with the Greek word Genesis (origin).

- WHO has for many years demanded for a reorientation in health care representing the use of both the resource (salutogenesis) and the treatment (pathogenesis) paradigms as complementary in health care. The Ottawa Charter became the answer to the request for a reorientation of the world's health care systems; there is still quite an unrealized potential in health care to be more protective and promotive of positive health.

- Applying salutogenesis as a health theory in the health promotion field could mean to restrict the leading pathogenic orientation in health care practice (research and policy) and complement or change it by a salutogenic orientation in everyday practice and research.

- Salutogenesis as a concept is understood to describe the process of enabling individuals, groups, organizations, and societies to emphasize abilities, resources, capacities, competences, strengths, and forces to create a sense of coherence; that is, to perceive life as comprehensible, manageable, and meaningful.

- The salutogenic model includes three central concepts: generalized resistance resources (GRRs), specific resistance resources (SRR), and sense of coherence (SOC).

- In health care settings, the salutogenic paradigm can be used for two purposes: either to guide health promotion interventions in health care practice across settings or to (re)orient health care research as such. For this, the salutogenic paradigm offers specific concepts, assumptions, and instruments.

\section{References}

1. Mittelmark MB, Sagy S, Eriksson M, Bauer GF, Pelikan JM, Lindström B, et al., editors. The handbook of Salutogenesis. New York: Springer; 2017.

2. WHO. The Ottawa charter for health promotion-World 1986. 1986. http://www.who.int/ healthpromotion/conferences/previous/ottawa/en/. 
3. Lindström B, Eriksson M. The Hitchhiker's guide to Salutogenesis. Folkhälsan Research Center, Health Promotion Research Programme and the IUHPE Global Working Group on Salutogenesis. ISBN: 978952-5641-34-9; 2010.

4. Antonovsky A. Unraveling the mystery of health: how people manage stress and stay well. San Francisco: Jossey-Bass; 1987.

5. Antonovsky A. The salutogenic model as a theory to guide health promotion. Health Promot Int. 1996;11(1):11-8.

6. Antonovsky A. The salutogenic approach to aging. Lecture held in Berkeley. January 21, 1993.

7. Antonovsky A. Health, stress, and coping: new perspectives on mental health and physical wellbeing. San Francisco, CA: Jossey-Bass; 1979.

8. De Leeuw E. Have the health services reoriented at all? Health Promot Int. 2009;24(2):105-7.

9. Wise M, Nutbeam D. Enabling health systems transformation: what progress has been made in reorienting health services? Promot Educ. 2007;2:23-7.

10. Pelikan JM. The application of Salutogenesis in healthcare settings. In: Mittelmark MB, Sagy S, Eriksson M, Bauer GF, Pelikan JM, Lindström $\mathrm{B}$, et al., editors. The handbook of Salutogenesis. New York: Springer; 2017.

11. Mittelmark M, Bull T. The salutogenic model of health in health promotion research. Glob Health Promot. 2013;20(2):30-8.

12. Lillefjell M, Magnus E, Knudtsen MS, Wist G, Horghagen S, Espnes GA, Maass REK, Anthun S. Governance for public health and health equity: The Trøndelag model for public health work. Scand J Public Health. 2018;46(22):37-47. https://doi. org/10.1177/1403494818765704.
13. Moksnes UK, Espnes GA. Sense of coherence in association with stress experience and health in adolescents. Int J Environ Res Public Health. 2020;17:3003.

14. Rinnan E, Andre B, Drageset J, Garåsen H, Espnes GA, Haugan G. Joy of life in nursing homes: a qualitative study of what constitutes the essence of joy of life in elderly individuals living in Norwegian nursing homes. Scand J Caring Sci. 2018;32(4):1468-76.

15. Seah B, Espnes GA, Ang ENK, Lim JY, Kowitlawakul Y, Wang W. Achieving healthy ageing through the perspective of sense of coherence among senior only households: a qualitative study. Aging Ment Health. 2020:1-10. https://doi.org/10.1080/13607863.2020.1 725805 .

16. Espnes GA. Salutogenesis: the editors discuss possible futures. In: Mittelmark MB, Sagy S, Eriksson M, Bauer GF, Pelikan JM, Lindström B, et al., editors. The handbook of Salutogenesis. New York: Springer; 2017.

17. Bauer GF, Roy M, Bakibinga P, Contu P, Downe S, Eriksson M, Espnes GA, Jensen BB, Canal DJ, Lingström B, Mana A, Mittelmark MB, Morgan AR, Pelikan JM, Saboga-Nunes L, Sagy S, Shorey S, Vandraager L, Vinje HF. Future directions for the concept of salutogenesis: a position article. Health Promot Int. 2020;35(2):187-95.

18. Dalton C, McCartney K. Salutogenesis: a new paradigm for pervasive computing in healthcare environments? In: Conference: 5 th international conference on pervasive computing technologies for healthcare, pervasive health, 2011, Dublin; 2011. https://doi. org/10.4108/icst.pervasivehealth.2011.246064.

Open Access This chapter is licensed under the terms of the Creative Commons Attribution 4.0 International License (http://creativecommons.org/licenses/by/4.0/), which permits use, sharing, adaptation, distribution and reproduction in any medium or format, as long as you give appropriate credit to the original author(s) and the source, provide a link to the Creative Commons license and indicate if changes were made.

The images or other third party material in this chapter are included in the chapter's Creative Commons license, unless indicated otherwise in a credit line to the material. If material is not included in the chapter's Creative Commons license and your intended use is not permitted by statutory regulation or exceeds the permitted use, you will need to obtain permission directly from the copyright holder. 